Investigation of the radiation hardness of GaAs sensors in an electron beam

This article has been downloaded from IOPscience. Please scroll down to see the full text article.

2012 JINST 7 P11022

(http://iopscience.iop.org/1748-0221/7/11/P11022)

View the table of contents for this issue, or go to the journal homepage for more

Download details:

IP Address: 141.34.3.153

The article was downloaded on 11/01/2013 at 16:00

Please note that terms and conditions apply. 


\section{Investigation of the radiation hardness of GaAs sensors in an electron beam}

\section{K. Afanaciev, ${ }^{a, 1}$ M. Bergholz, ${ }^{b}$ P. Bernitt, ${ }^{b, 2}$ G. Chelkov, ${ }^{c}$ J. Gajewski, ${ }^{d}$ M. Gostkin, ${ }^{c}$ Ch. Grah, ${ }^{e, 3}$ R. Heller, ${ }^{e}$ H. Henschel, ${ }^{e}$ A. Ignatenko, ${ }^{a, b, e}$ Z. Krumshteyn, ${ }^{c}$ S. Kulis, ${ }^{d}$ W. Lange, ${ }^{e}$ W. Lohmann, ${ }^{b, e}$ D. Mokeev, ${ }^{f}$ V. Novikov, ${ }^{f}$ M. Ohlerich, $,{ }^{b, e}, 4$ A. Rosca,,${ }^{g, e}$} A. Sapronov, ${ }^{c}$ R.S. Schmidt, ${ }^{b, e}$ S. Schuwalow, ${ }^{e, 5}$ O. Tolbanov ${ }^{f}$ and A. Tyazhev ${ }^{f}$ ${ }^{a}$ NC PHEP BSU, Minsk, Belarus

${ }^{b}$ Brandenburgische Technische Universität Cottbus, Cottbus, Germany

${ }^{c}$ Joint Institute for Nuclear Research, Dubna, Russia

${ }^{d}$ AGH University of Cracow, Cracow, Poland

${ }^{e}$ Deutsches Elektronen-Synchrotron, Germany

${ }^{f}$ Tomsk State University, Tomsk, Russia

${ }^{g}$ West University of Timisoara, Timisoara, Romania

E-mail: akg@ifh.de

ABSTRACT: A compact and finely grained sandwich calorimeter is designed to instrument the very forward region of a detector at a future $\mathrm{e}^{+} \mathrm{e}^{-}$collider. The calorimeter will be exposed to low energy $\mathrm{e}^{+} \mathrm{e}^{-}$pairs originating from beamstrahlung, resulting in absorbed doses of about one MGy per year. GaAs pad sensors interleaved with tungsten absorber plates are considered as an option for this calorimeter. Several Cr-doped GaAs sensor prototypes were produced and irradiated with $8.5-10 \mathrm{MeV}$ electrons up to a dose of $1.5 \mathrm{MGy}$. The sensor performance was measured as a function of the absorbed dose.

KEYWORDS: Radiation-hard detectors; Radiation damage to detector materials (solid state); Calorimeters

\footnotetext{
${ }^{1}$ Corresponding author.

${ }^{2}$ Now at Chalmers University of Technology, Gothenburg, Sweden.

${ }^{3}$ Now at BTO Management Consulting AG, Berlin, Germany.

${ }^{4}$ Now at Technische Universität München, München, Germany.

${ }^{5}$ Now at Universität Hamburg, Hamburg, Germany.
} 


\section{Contents}

1 Introduction 1

2 The gallium arsenide sensors $\quad 2$

3 Measurements before irradiation 3

3.1 Current-voltage measurements 3

3.2 Capacitance-voltage measurements 4

3.3 Charge collection efficiency measurement 5

4 Irradiation setup $\quad 6$

5 Measurements at the time of irradiation $\quad 8$

6 Measurements after irradiation $\quad 9$

7 Conclusion 9

\section{Introduction}

Detectors in a future high energy electron-positron collider will face a new phenomenon at small polar angles - a large amount of low energy electron-positron pairs originating from beamstrahlung [1]. A few percent of the beam energy is radiated as photons escaping inside the beampipe. However, a fraction of these photons converts into electron-positron pairs that are deflected to larger polar angles. These particles hit the detectors adjacent to the beam-pipe. In the current detector concepts [2] a calorimeter, BeamCal, is foreseen at small polar angles. BeamCal will assist beam tuning by measuring the deposition of beamstrahlung pairs as well as veto single high energy electrons. The latter is mandatory to suppress background from two-photon processes in new particle searches. To avoid uninstrumented regions and due to a limited space no cooling is foreseen. The device will be operated at room temperature and the front-end electronics will be operated in a power switching mode.

A compact and finely segmented cylindrical sandwich calorimeter is designed [3]. Tungsten disks of approximately one radiation length thickness serve as absorber layers and are interspersed with thin sensor layers with pad segmentation. Monte-Carlo simulations have shown that with pad sizes below the Moliere radius of about $1 \mathrm{~cm}$, e.g. $5 \times 5 \mathrm{~mm}^{2}$, the necessary performance will be reached. Sensor pads adjacent to the beampipe are irradiated with a dose of a MGy per year of operation due to the depositions of low energy pairs from beamstrahlung. The energy of these pairs ranges from a few $\mathrm{keV}$ up to several $\mathrm{GeV}$. The largest contribution to the energy deposition in the calorimeter results from shower particles with an energy in the MeV range. An absorbed dose of 
Table 1: Material properties.

\begin{tabular}{|c|c|c|c|c|c|}
\hline & Sample & Donor & $\begin{array}{c}\text { Donor concentration } \\
\mathrm{cm}^{-3}\end{array}$ & Acceptor & $\begin{array}{c}\text { Thickness } \\
\mu \mathrm{m}\end{array}$ \\
\hline Lot 0 & GaAs1, GaAs2 & $\mathrm{Te}$ & $(5-6) \cdot 10^{16}$ & $\mathrm{Cr}$ & 500 \\
Lot 1 & 9,11 & $\mathrm{Te}$ & $(1-1.5) \cdot 10^{17}$ & $\mathrm{Cr}$ & 170 \\
Lot 2 & 5,7 & $\mathrm{Te}$ & $(5-6) \cdot 10^{16}$ & $\mathrm{Cr}$ & 190 \\
Lot 3 & 2,31 & $\mathrm{Sn}$ & $(1-3) \cdot 10^{16}$ & $\mathrm{Cr}$ & 160 \\
\hline
\end{tabular}

$1 \mathrm{MGy}$ for a GaAs detector corresponds to a fluence of approximately $1,5 \cdot 10^{15} 10 \mathrm{MeV}$ electrons per $\mathrm{cm}^{2}$.

Silicon sensors are not sufficiently radiation hard at room temperature due to the dramatic rise of the leakage current [4]. The results of an irradiation study of a silicon sensor at similar irradiation conditions are described in [5].

Sensors made of Cr-doped GaAs are an option for BeamCal because of their expected radiation hardness. Studies on radiation hardness of semi-insulating GaAs detectors were done previously in the framework of the CERN RD8 research program. It was found that GaAs sensors could withstand fluences of $3 \cdot 10^{14}$ protons, $2 \cdot 10^{14}$ pions and $6 \cdot 10^{14}$ neutrons per $\mathrm{cm}^{2}$ for undoped GaAs [6]. For compensated GaAs sensors the reported radiation hardness for hadronic irradiation is of the same order of magnitude [7]. Irradiations with $0.66 \mathrm{MeV}$ photons were carried out up to doses of $100 \mathrm{kGy}$ and have shown a slight decrease in the detector efficiency [7]. Results obtained with 1.17 and $1.33 \mathrm{MeV}$ photons from a ${ }^{60} \mathrm{Co}$ source did not show any significant damage up to doses of 1 MGy [8].

The goal of this study is a measurement of the radiation hardness of compensated semiinsulating GaAs detector material for irradiation with electrons. GaAs sensors manufactured with different concentrations of dopants were exposed to electron beams of 8.5 and $10 \mathrm{MeV}$ energy. The charge collection efficiency (CCE) was measured as a function of the absorbed dose. The maximum accumulated dose for a sensor was $1.5 \mathrm{MGy}$. Sensors were kept under bias voltage without interruption during irradiation and CCE measurements. The leakage current was measured as a function of the applied voltage before and after irradiation.

\section{The gallium arsenide sensors}

The GaAs sensors investigated were produced by means of the Liquid Encapsulated Czochralski (LEC) method. The initial LEC GaAs material is doped by a shallow donor ( $\mathrm{Sn}$ or Te). This doping is necessary to form a low-ohmic n-type semiconductor and to fill EL+ trapping centers with electrons. Then the material is compensated by a deep acceptor $(\mathrm{Cr})$ by means of controlled diffusion at high temperature [9-11]. This results in a semi-insulating GaAs material with a resistivity of about $10^{7} \Omega \mathrm{m}$ [10]. The samples used here are subdivided into 4 production lots differing in type and concentration of the shallow donor. The sample properties are listed in table 1 .

It should be noted that, due to the properties of the compensated material, the hole lifetime is very low and the signal is predominantly generated by electron transport. As a consequence, the 


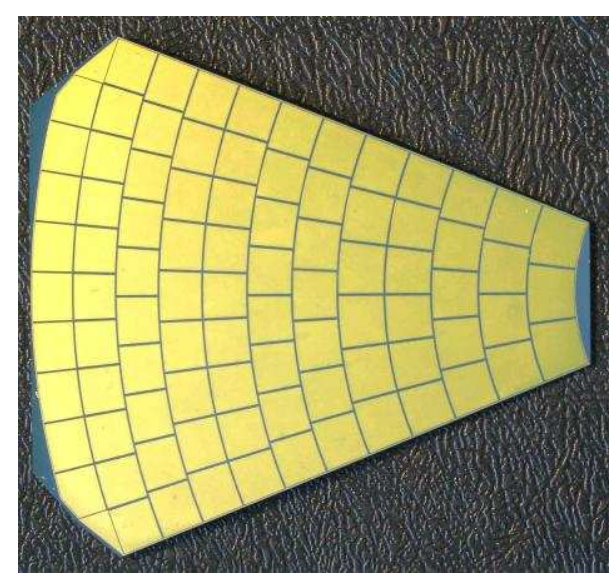

(a)

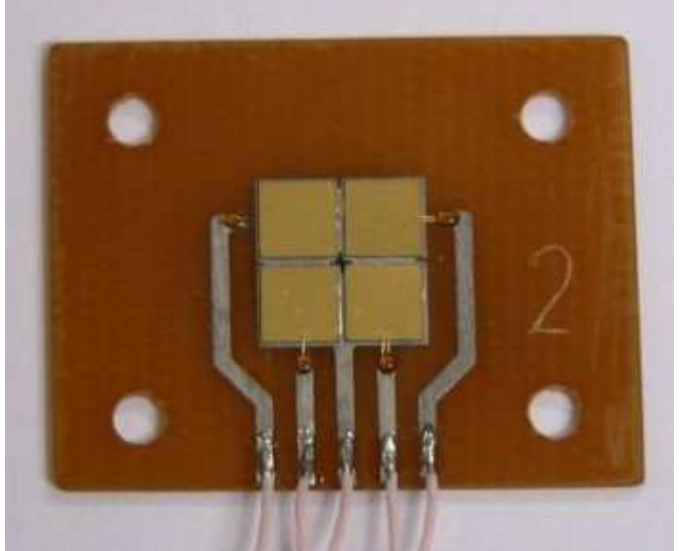

(b)

Figure 1: GaAs sensors for a BeamCal prototype: Layout 1(a) - lot 0 . A gallium arsenide plate has a shape of a $45^{\circ}$ sector. The metallisation layer is subdivided into 12 ring segments with innermost radius $20 \mathrm{~mm}$ and outermost radius $84 \mathrm{~mm}$. Each ring segment is divided into pads of approximately $5 \times 5 \mathrm{~mm}^{2}$ size. Layout 2 (b) - lots 1,2 and 3. Four separate sensor pads of $5 \times 5 \mathrm{~mm}^{2}$ each, combined into a square sensor.

maximum reachable charge collection efficiency of the detector is $50 \%$ for particles fully crossing the sensor. This feature is explained in detail in [12]. The signal formation due to electron transport only was confirmed by measurements of the signal size for different high voltage polarities using an $\alpha$-source.

Two different layouts of sensors were investigated. The first one is shown in figure 1(a). It was processed from material of lot 0 . The sensor is $500 \mu \mathrm{m}$ thick. Two sensors of this type, hereafter referred to as GaAs1 and GaAs2, were investigated. The second layout is a composite sensor consisting of four individual sensor pads arranged in a square as shown in figure 1(b). Sensors from different lots have different thicknesses as shown in table 1. Six such sensors, two from each of the lots 1, 2 and 3 were investigated. For all sensors from all lots the metallisation consists of a $1 \mu \mathrm{m}$ gold layer on top of a $30 \mathrm{~nm}$ vanadium layer on both sides.

\section{Measurements before irradiation}

\subsection{Current-voltage measurements}

For all sensors the current as a function of the applied voltage (I-V characteristic) was measured.

An I-V characteristic for a pad of sample GaAs2 of lot 0 is shown in figure 2(a). The currents are of the order of $0.45 \mu \mathrm{A}$ for a field strength of $0.4 \mathrm{~V} / \mu \mathrm{m}$ which corresponds to a bias voltage of $200 \mathrm{~V}$. All pads of both sensors from lot 0 show similar I-V characteristics within 5\%. The I-V curves are symmetric for both polarities.

The I-V curve is slightly asymmetric for samples from lot 1 as shown in figure 2(b). The currents are of the order of $0.1 \mu \mathrm{A}$ for a field strength of $0.4 \mathrm{~V} / \mu \mathrm{m}$. The I-V curve of a sample from lot 2 is shown in figure 2(c). It also has a slight asymmetry and the current value at $0.4 \mathrm{~V} / \mu \mathrm{m}$ field strength is close to that of the samples from lot 1. 


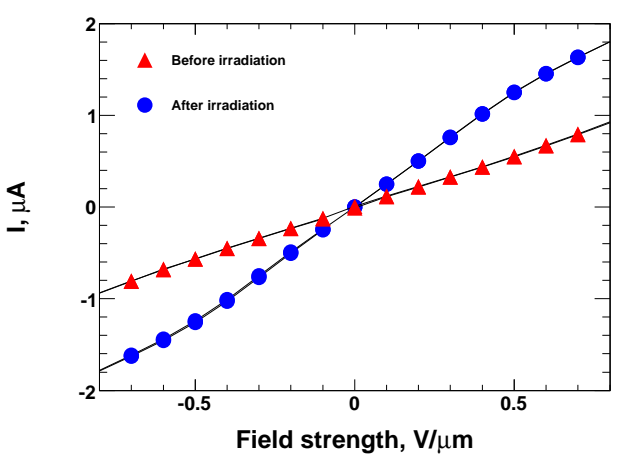

(a)

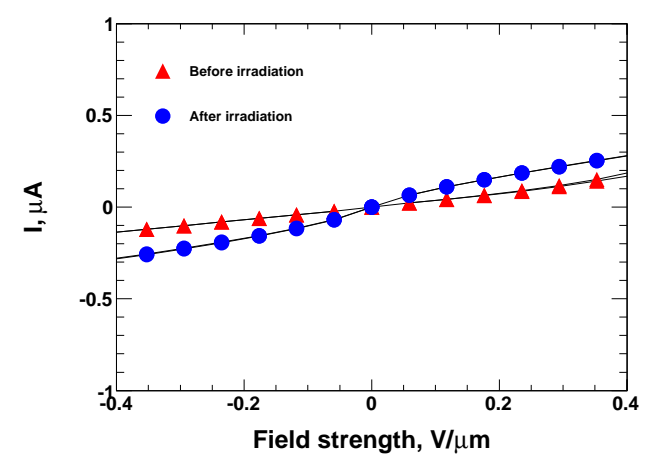

(c)

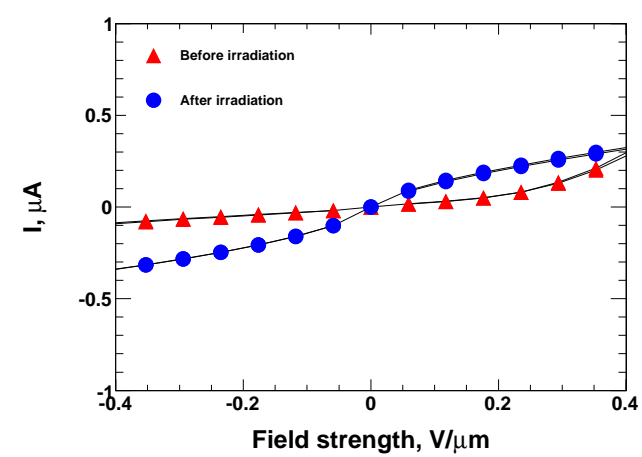

(b)

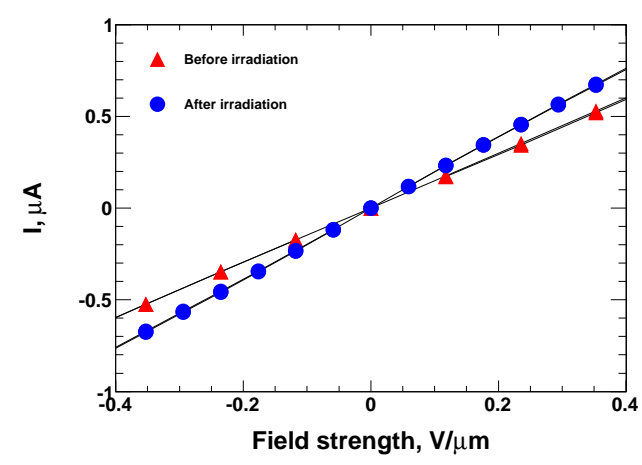

(d)

Figure 2: The current-voltage characteristics of GaAs sensors before and after irradiation: a sensor from lot 0 (a), lot 1 (b), lot 2 (c) and lot 3 (d). The voltage is normalized to the sensor thickness.

The I-V curve of a sample from lot 3 is shown in figure 2(d). It is similar to the one from the sensors from lot 0 . The sensors from lot 1 have the lowest currents whereas the sensors from lot 3 have the highest currents at the same field strength.

\subsection{Capacitance-voltage measurements}

The capacitance of the pads for sensors from lot 0 is approximately $13 \mathrm{pF}$. The capacitance of individual pads is in the range $15-17 \mathrm{pF}$ for the samples from lots 1,2 and 3.

For selected unirradiated and irradiated pads of sensors from lots 1,2 and 3 the capacitance as a function of the applied voltage ( $\mathrm{C}-\mathrm{V}$ characteristic) was measured for several frequencies $(120 \mathrm{~Hz}, 1 \mathrm{kHz}, 10 \mathrm{kHz}, 100 \mathrm{kHz}$ and $1 \mathrm{MHz})$. The individual pads of the investigated samples were contacted with needle probes and the bias voltage was applied through a coupling box. The measurements were done in a shielded light-tight box.

An example of a C-V characteristic for different frequencies is shown in figure 3. The C-V dependence is linear for higher frequencies and shows some nonlinearity at lower frequencies for both unirradiated and irradiated pads. The largest difference in capacitance between unirradiated and irradiated pads is in the order of $10 \%$. The capacitance of the pads at higher frequencies is close to the calculated value for a parallel plate capacitor. 


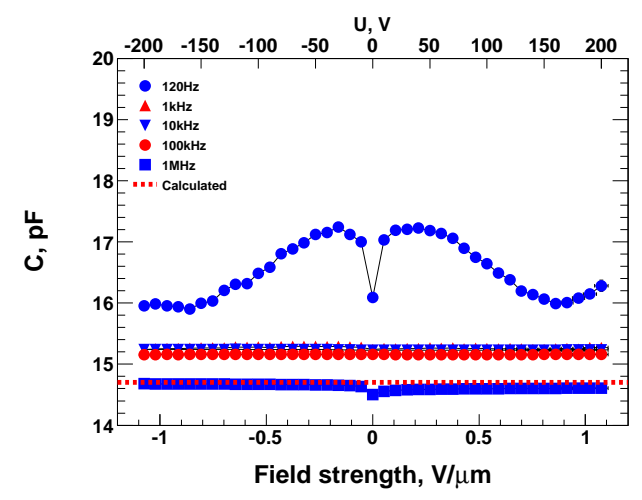

(a)

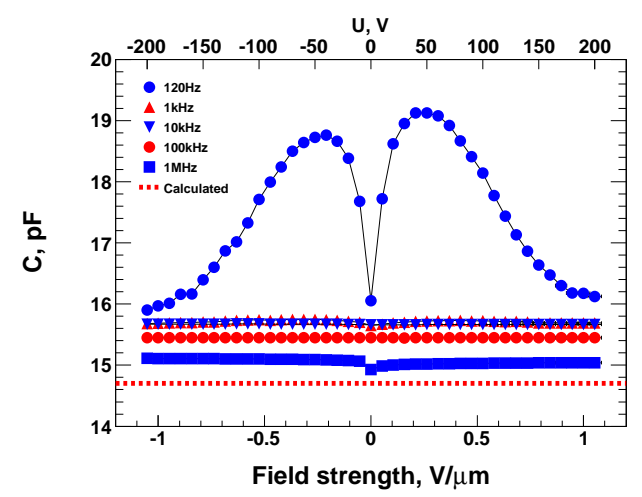

(b)

Figure 3: Capacitance as a function of voltage for different frequencies. Sample 5 (Lot 2) - (a) unirradiated pad, (b) pad irradiated to a dose of $820 \mathrm{kGy}$.

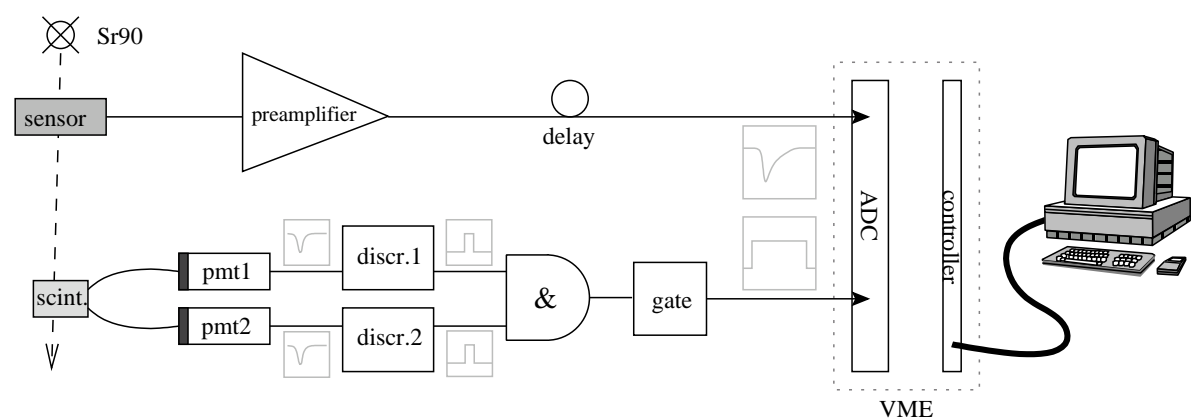

Figure 4: Diagram of the setup for the measurement of the response of a sensor to charged particles. An electron from a ${ }^{90} \mathrm{Sr} \beta$ source traverses the sensor and generates a signal in the scintillator below. The two photomultipliers generate a trigger signal and the charge sensitive ADC digitizes the amplified and shaped signal from the sensor.

\subsection{Charge collection efficiency measurement}

The charge collection efficiency (CCE) as a function of the applied voltage was measured for selected pads.

The diagram of the setup used for the charge collection efficiency (CCE) measurement is shown in figure 4. Signals from the sensor are read out with a charge-sensitive amplifier and then digitized by a gated charge integrating ADC. The readout chain was calibrated by injection of a known charge at the amplifier input, thus allowing for the conversion of measured ADC data into charge units.

The charge collection efficiency is defined as: $C C E=Q_{\text {measured }} / Q_{\text {generated }}$ where $\mathrm{Q}_{\text {measured }}$ is the measured charge and $\mathrm{Q}_{\text {generated }}$ is the expected charge to be produced by ionization from a particle.

A scintillator triggers on electrons that have passed through the sensor under investigation. The scintillator is read out by two photomultipliers operating in coincidence. From a MonteCarlo simulation the energy spectrum of the electrons generating a trigger was obtained. For these 


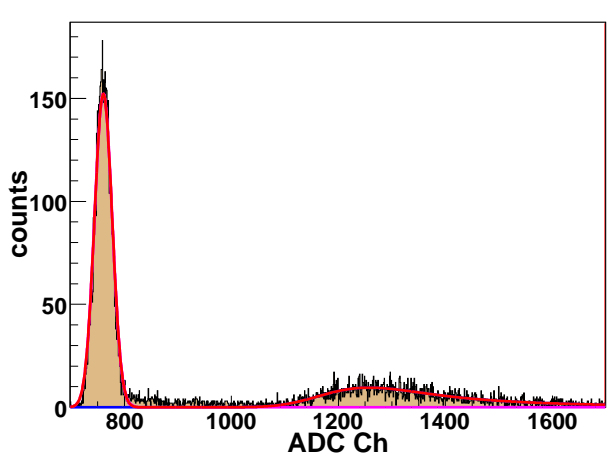

(a)

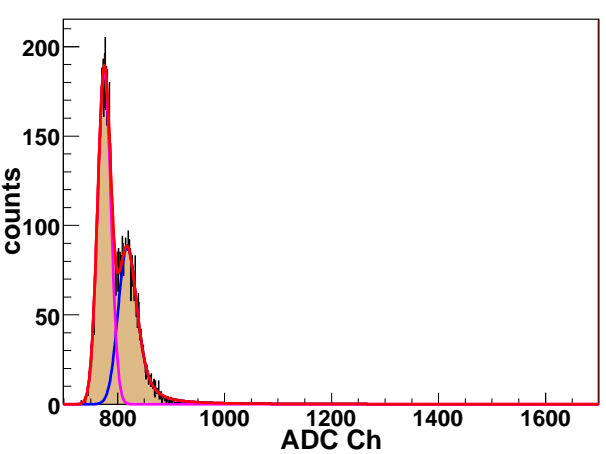

(b)

Figure 5: Spectrum from an unirradiated sensor GaAs2 (a) and from the same sensor after absorbing a dose of $1.5 \mathrm{MGy}(\mathrm{b})$.

electrons the energy loss in GaAs was calculated and used in the calculation of the expected charge

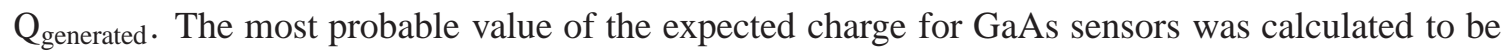
150 electron-hole pairs per $\mu \mathrm{m}$. The calculations were based on a GEANT 4 simulation of the experimental setup and the value of the energy needed to create an electron-hole pair in GaAs [13].

$\mathrm{Q}_{\text {measured }}$ is obtained by measuring the response of the detector to electrons from a ${ }^{90} \mathrm{Sr}$ source. It is derived from a fit of the corresponding signal spectrum as shown in figure 5(a). The pedestal peak on the left is described by a Gaussian and the signal spectrum on the right by a Landau distribution convoluted with a Gaussian. The difference between the most probable value of the Gauss-Landau distribution and the mean value of the pedestal is taken as signal value.

A signal spectrum obtained from a pad of a non-irradiated sensor is shown in figure 5(a). A clear separation between the pedestal peak and signals of crossing electrons is observed.

The CCE as a function of the bias voltage for one sample of lot 0 is shown in figure 6. At low voltages the CCE rises and then reaches a saturation. The saturation appears at voltages of around $200 \mathrm{~V}$ for sensors from lot 0 which corresponds to an electric field strength of $0.4 \mathrm{~V} / \mu \mathrm{m}$. For samples from lots 1,2 and 3 the saturation appears at $35-40 \mathrm{~V}(0.2 \mathrm{~V} / \mu \mathrm{m})$. The saturated CCE values are about $50 \%$ for samples from lots 0 and 3, about $40 \%$ for lot 2 and $30 \%$ for lot 1 .

\section{Irradiation setup}

An electron beam of up to $50 \mathrm{nA}$ beam current at the 'Superconducting Darmstadt Linear Accelerator', S-DALINAC [14], was used to irradiate the samples. A beam energy of $10 \mathrm{MeV}$ was chosen for the irradiation. It is comparable to the expected energies of the shower particles originating from the beamstrahlung-generated electrons in the BeamCal.

Samples from lot 0 were irradiated with a $10 \mathrm{MeV}$ beam and samples from lots 1,2 and 3 were irradiated with a $8.5 \mathrm{MeV}$ beam. ${ }^{1}$

\footnotetext{
${ }^{1}$ Due to change in the technical conditions.
} 


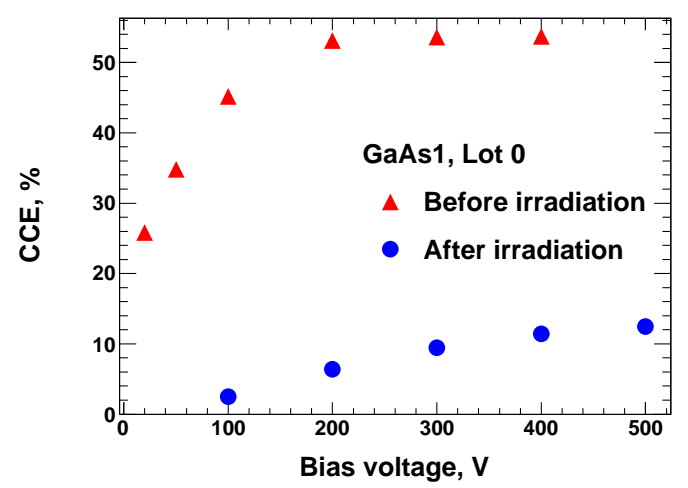

Figure 6: The CCE as a function of the applied voltage for a pad of a sensor from Lot 0. Measurements before and after the irradiation.

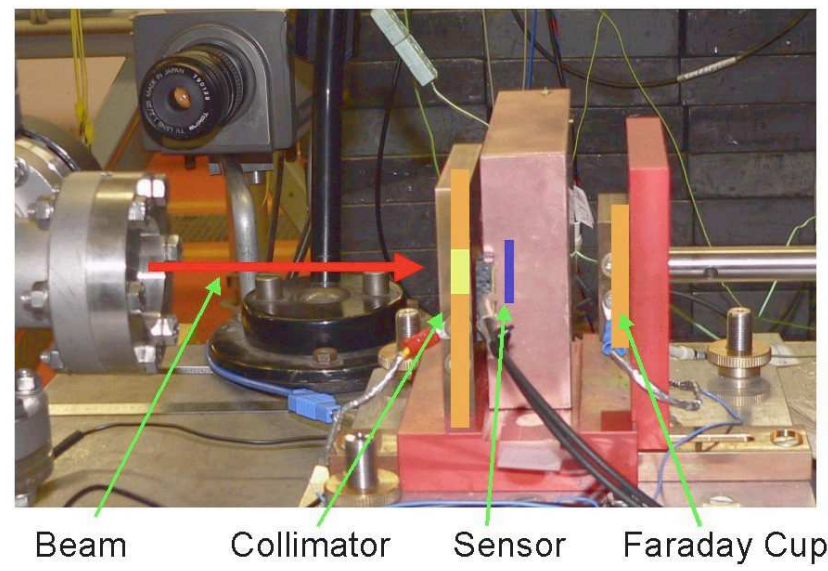

Figure 7: Experimental setup for sensor irradiation. The electron beam exit window is shown on the left. The transverse beam size is determined by a copper collimator. The sensor under test is positioned in an electrically screened box. The Faraday cup is used to measure the current of the beam crossing the sensor.

The electron transport through the irradiation setup was simulated with GEANT4 to estimate corrections for backscattering of electrons from the Faraday cup and to determine the energy deposited in the sensor by the beam [15].

The irradiation setup is shown in figure 7. The electron beam comes from the left as indicated by the red arrow. The beam is scattered by the aluminum exit window inside the flange. The beam profile is shaped by a collimator of $10 \mathrm{~mm}$ thick copper with a square aperture of $9 \times 9 \mathrm{~mm}^{2}$. The beam crosses the sensor which is mounted in an electrically screened box and is then absorbed in a Faraday cup made of a $10 \mathrm{~mm}$ thick copper plate of $40 \times 40 \mathrm{~mm}^{2}$ in size. This allows the measurement of the beam current through the sensor. The sensor is aligned to the beam such that the center of the pad selected for the irradiation is in the center of the beam line. Since the size of the beamspot was larger than a pad, the neighbor pads were partly irradiated as well.

During the measurements the beam current was varied between $2.5 \mathrm{nA}$ and $50 \mathrm{nA}$, corresponding to dose rates in the sensor from $20 \mathrm{kGy} / \mathrm{h}$ to $400 \mathrm{kGy} / \mathrm{h}$. 


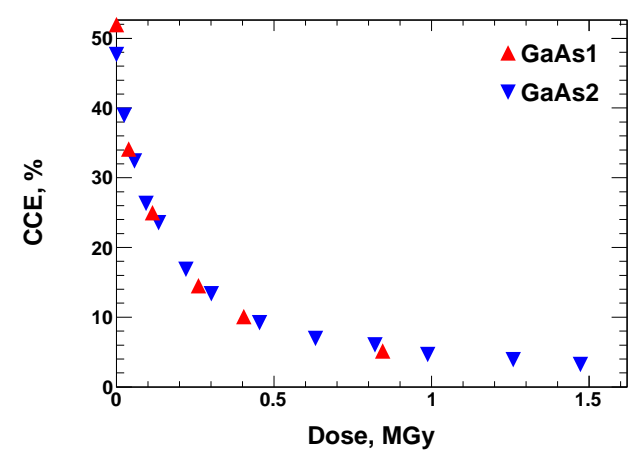

(a)

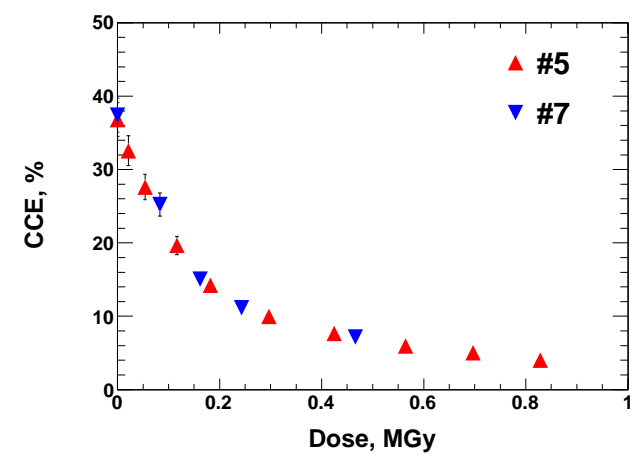

(c)

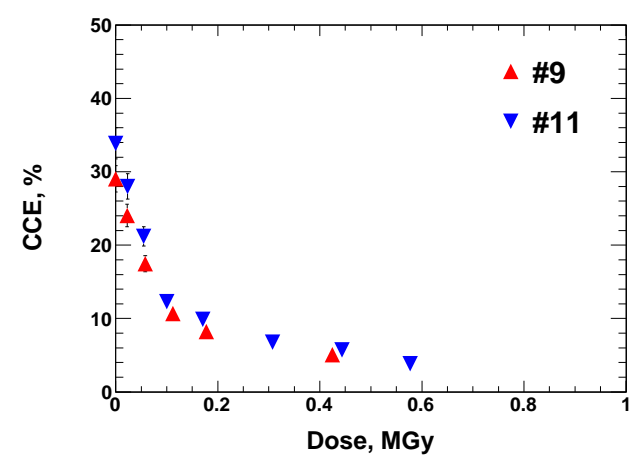

(b)

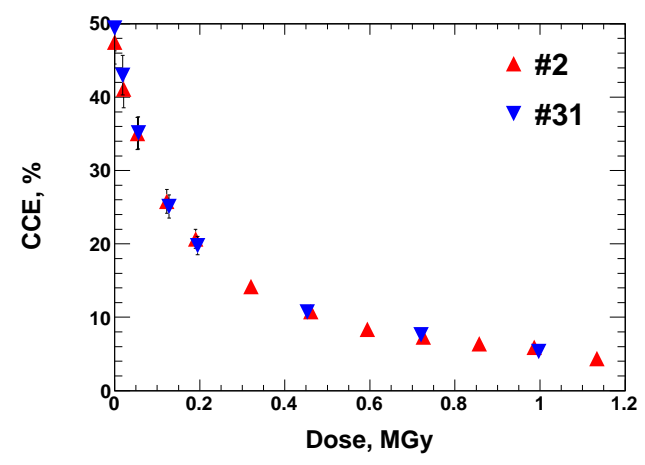

(d)

Figure 8: The CCE as a function of the absorbed dose for the sensors from Lot 0 (a), Lot 1 (b), Lot 2 (c) and Lot 3 (d).

The sensors were kept under bias voltage for the whole duration of the measurements. The bias voltage was $200 \mathrm{~V}$ for sensors from lot 0 corresponding to an electric field strength of $0.4 \mathrm{~V} / \mu \mathrm{m}$ and $40 \mathrm{~V}$ for sensors from lots 1,2 and $3(0.2 \mathrm{~V} / \mu \mathrm{m})$. In intervals of approximately one hour the beam was stopped and the sensor box with the biased sensor was taken to a setup as shown in figure 4 to measure the $\mathrm{CCE}$ and the leakage current of the pad under investigation. These measurements took about 30 minutes and then the irradiation was continued.

The resulting irradiation rate by the ${ }^{90} \mathrm{Sr}$ source of the CCE measurement setup was about $0.05 \mathrm{~Gy} / \mathrm{h}$, hence negligible in comparison to the irradiation by the accelerator beam.

\section{Measurements at the time of irradiation}

In figure 8(a) the CCE as a function of the absorbed dose is shown for both sensor samples of lot 0 . The degradation of the collection efficiency with the absorbed dose is similar for both samples.

The CCE dependence on the absorbed dose for the sensors from lots 1, 2 and 3 is shown in figure 8(b), (c) and (d), respectively. Figure 9 represents the dependence for sensors from 4 different lots; the CCE shown here is normalized to the initial value of the CCE of the corresponding sensor before irradiation. The CCE of the sensors from the lots 0 and 3 is the highest before irradiation and its degradation with the absorbed dose is slower. Sensors from lot 1 had the lowest initial CCE 


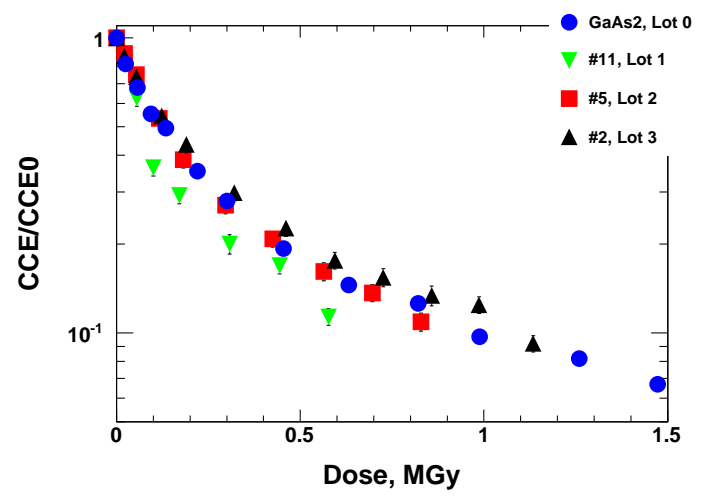

Figure 9: The CCE as a function of the absorbed dose. CCE is normalized to initial CCE of the sample and plotted on a logarithmic scale. The samples represent all material lots.

and the CCE degradation during irradiation was more pronounced. Sensors from lot 2 have shown intermediate results both in initial CCE value and in CCE degradation with absorbed dose.

\section{Measurements after irradiation}

After the irradiation the I-V characteristics and CCE were measured for all sensor samples in the laboratory. The leakage currents for sensors from lot 0 increased after irradiation approximately by a factor of two as can be seen in figure 2(a). The shapes of I-V curves for sensors of lots 1 and 2 became more symmetric as it is shown in figure 2(b) and (c). The increase in the leakage currents was about a factor of 3 for lot 1 , about a factor of 2 for lot 2 and 1.5 for lot 3 .

For the samples from lot 0 the values of the CCE decreased after irradiation to less than $10 \%$ of the value before irradiation as can be seen in figure 9. The saturation of the signal value appeared at about $200 \mathrm{~V}$ bias voltage for unirradiated samples. After the irradiation the saturation voltage increased significantly as shown in figure 6 . The difference in the signal spectra from the ${ }^{90} \mathrm{Sr}$ source is shown in figure 5 . The capacitance of the samples remained unchanged after the irradiation.

\section{Conclusion}

Several Cr-doped GaAs sensor samples have been investigated. The I-V measurements show that after an irradiation with doses of up to $1.5 \mathrm{MGy}$ the leakage currents do not increase at room temperature by more than a factor of three at an operational electric field strength between 0.2 and $0.4 \mathrm{~V} / \mu \mathrm{m}$.

The CCE as a function of the bias voltage saturates at higher voltages. The saturated value of the CCE reaches about $50 \%$ for unirradiated samples. For irradiated samples no saturation is observed for field strengths up to $1 \mathrm{~V} / \mu \mathrm{m}$.

The CCE drops with absorbed dose more rapidly at low doses. The CCE is reduced to about $10 \%$ of the initial value after a dose of $1 \mathrm{MGy}$.

The results indicate that there is a possible dependence of the radiation hardness on the type and concentration of the shallow donor. The samples doped with the same type of shallow donor 
(Te) have better radiation hardness at lower dopant concentrations. The samples doped with Sn at low concentration also have a better radiation hardness, correspondingly.

The signals of minimum-ionising particles are still separated from the noise after a dose of $1 \mathrm{MGy}$, allowing a continuous in-situ calibration of the gain of the sensor pads using abundant muons from beam-halo. Hence we consider the results on the radiation tolerance of GaAs sensors under electron radiation as very promising for the feasibility of a BeamCal instrumented with GaAs sensors.

\section{Acknowledgments}

We are grateful to the crew and the management of the S-DALINAC accelerator at the Technical University of Darmstadt for their great support during the measurements at the electron beam. We would also like to thank "The International Association for the promotion of Co-operation with Scientists from the New Independent States of the former Soviet Union", INTAS, for the support within the Young Scientists grants, and the World Laboratory for awarding a Bjoern Wiik fellowship.

\section{References}

[1] D. Schulte, Study of electromagnetic and hadronic background in the interaction region of the TESLA Collider, Ph.D. Thesis, Universität Hamburg, Hamburg Germany (1996).

[2] International Linear Collider, ILC reference design report. Volume 4-Detectors, http://www.linearcollider.org/about/Publications/Reference-Design-Report.

[3] H. Abramowicz et al., Forward instrumentation for ILC detectors, 2010 JINST 5 P12002.

[4] G. Lindström, M. Moll and E. Fretwurst, Radiation hardness of silicon detectors - a challenge from high-energy physics, Nucl. Instrum. Meth. A 426 (1999) 1.

[5] C. Grah et al., Polycrystalline CVD diamonds for the beam calorimeter of the ILC, IEEE Trans. Nucl. Sci. 56 (2009) 462.

[6] RD8 collaboration, R.L. Bates et al., The effects of radiation on gallium arsenide radiation detectors, Nucl. Instrum. Meth. A 395 (1997) 54.

[7] V. Chmill, Radiation tests of semiconductor detectors, Ph.D. Thesis, Stockholm Sweden (2006).

[8] T. Ly Anh, A. Perd'ochová, V. Nečas and V. Pavlicová, Radiation resistance study of semi-insulating GaAs-based radiation detectors to extremely high gamma doses, Nucl. Phys. Proc. Suppl. B 150 (2006) 402.

[9] G.I. Ayzenshtat et al., GaAs structures for X-ray imaging detectors, Nucl. Instrum. Meth. A 466 (2001) 25.

[10] G.I. Ayzenshtat et al., GaAs as a material for particle detectors, Nucl. Instrum. Meth. A 494 (2002) 120.

[11] A.V. Tyazhev et al., GaAs radiation imaging detectors with an active layer thickness up to $1 \mathrm{~mm}$, Nucl. Instrum. Meth. A 509 (2003) 34. 
[12] G.I. Ayzenshtat et al., GaAs resistor structures for X-ray imaging detectors, Nucl. Instrum. Meth. A 487 (2002) 96.

[13] J.E. Eberhardt, R.D. Ryan and A.J. Tavendale, Evaluation of epitaxial $n$-GaAs for nuclear radiation detection, Nucl. Instrum. Meth. 94 (1971) 463.

[14] Der supraleitende Darmstädter Elektronenbeschleuniger S-DALINAC, http://www.ikp.tu-darmstadt.de/sdalinac $j$ kp/.

[15] R. Schmidt, Optimisation of a testbeam setup and background estimates for detectors at the ILC using Monte Carlo simulations, Diploma Thesis, Brandenburgische Technische Universität Cottbus, Cottbus Germany (2007). 